\title{
A FURTHER ACCOUNT
}

or

\section{FATTY DEGENERATION OF THE PLACENTA,}

AND THE

INFLUENCE OF THIS DISEASE IN PRODUCING

DEATH OF THE FETUS, HAMORRHAGE, AND ABORTION.

BY

ROBERT BARNES, M.D. (LOND.),

OBSTETRIC SURGEON TO THE WESTERN GENERAL DISPENSARY, LECTURER ON MIDWIFERY AT THE ROYAL FREE HOSPITAL.

COMMUNICATED BY

PROFESSOR MURPHY.

Received Jan. 11th.--Read Feb. 22d, 1853.

Since the publication of my first paper and appendix in the 34th volume of the 'Medico-Chirurgical Transactions,' I have had the opportunity of examining several additional examples of fatty degeneration of the placenta. Some of these cases exhibit forms of this affection sufficiently distinct from the form described in that paper to merit a separate notice. Several present a form which corresponds closely with the description of Dr. Franz Kilian's case, ('Neue Zeitschrift für Geburtskunde,' vol. xxvii.) One case shows the co-existence of fatty degeneration, with hydatiginous degeneration of the chorion. Two are examples of fatty. degeneration of the chorion at a very early period, associated with abortion. 
The histories of the cases now submitted to the Society, viewed individually and collectively, will, I believe, be found to throw much additional light upon the pathology of fatty degeneration in an organ in which the existence of that affection has only recently been established. They lend confirmation also to the opinion I have before expressed, as to the importance of this affection as a cause of death of the fœtus, hæmorrhage, and abortion; and they illustrate the modes by which these events are brought about.

I am again indebted to my friend, Dr. Hassall, for his valuable aid in the microscopical investigations, and in the preparation of the pictorial delineations of the diseased structures. I have also to thank many gentlemen, to some of whom I was personally unknown, for having placed many interesting specimens of diseased ova at my disposal.

In numbering the cases I have thought it useful, having regard to future reference, to number them consecutively from those which form the subject of my first paper. These were three in number; the first case in this communication will therefore be numbered as the fourth.

Case IV.-On the 26th April, 1852, Mr. Humby, to whom I am indebted for the two placentas which first led me to the observation of fatty disease of the placenta, left me a placenta with the fœtus attached. The fœtus had appa. rently attained the middle period of gestation. It was shrunken, as if it had undergone maceration. The cuticle did not peel off; it was of a brownish colour. The cord had a similar appearance. The fœtus and cord were quite free from putrefaction. The lady who was the subject of this case had not been conscious of the death of the fœtus. She was taken suddenly with slight pains. Mr. Humby arrived in time to observe the passage of the child and placenta, which all came together. There had been some offensive discharge before labour; but no hæmorrhage occurred either preceding, during, or after the labour.

The placenta, like the child, was free from putrefaction. The maternal surface was deeply divided by sulci. Its general 
aspect much resembled that of the brain, both in colour and subdivision. All the lobes had a similar appearance. Pale, yellow, glistening like fat on the prominent portions; pink or red in the sulci. In the centre of some five or six of the lobes was a small dark clot, marking the recent rupture of a vessel connecting the uterus and the placenta. In the sulci between the lobes there was evidence of somewhat more extensive vascular connection with the uterus. With these exceptions, the placenta had generally an exsanguine appearance. A section showed that the yellow fatty appearance was most marked on the decidual surface; on approaching the fotal surface there was more appearance of blood. There was nothing like fresh healthy placental tissue. The consistence was very firm; it rather broke than tore, and cut like a solid body.

The villi taken from any part near the decidual surface were much altered; villi from nearer the fotal surface were less altered, but none were found perfectly healthy. The villi in the more consolidated lobes showed the greatest amount of alteration: they were brittle, misshapen; the vessels breaking up, and losing their defined outline and form ; the chorion was for the most part destroyed, but the nuclei in the walls of the vessels in many places were not, as is usually the case, destroyed, but enlarged and crammed with granules. In the less consolidated lobes of this placenta, the vessels retained their proper size and shape, but the chorion was destroyed to a great extent; the nuclei of the walls of the vessels had disappeared; numerous oilgranules were scattered over the walls, and in addition there were numerous masses of fatty matter, consisting of aggregations of globules, disposed usually in the course of the vessels, but sometimes confined to the extremities.

The following appears to me to be a rational exposition of the pathology of this case. The fœtus evidently perished some time before its expulsion. All fœtal-placental circulation had therefore ceased. The large vessels which still connected the placenta with the uterus were the channels by which an imperfect utero-placental circulation was carried on xXXvi. 
up to the last. As there could have been no interchange between maternal and fœetal blood, this circulation must have been simply to maintain a low degree of nutrition in the placenta. It is reasonable to infer that the granular degeneration had commenced prior to the death of the fotus; that its gradual advance having rendered the villi unfit for the interchange of the elements of the maternal and fœtal blood, had thus destroyed the foetus. A certain connection between the uterus and placenta was still kept up. The granular conversion of the villi continued to increase after the death of the fotus, until it had reached such an extent as to cut off so much of the remaining vascular communications as to leave the longer attachment of the placenta to the uterus impossible.

This gradual arrest of the circulation in the placenta, and the gradual obliteration of the utero-placental vessels before the detachment of the placenta, will explain the fact, that there was no hæmorrhage at the time of labour.

CASE v.-Mrs. R-, a patient of Mr. Barker, was taken in labour on the 25th of October, 1851. Considerable hæmorrhage in gushes occurred at the commencement. Mr. B. found the placenta at the os uteri partially detached. The funis was prolapsed. He aided the expulsion of the fotus. The placenta was partially adherent, so that it was extracted with some difficulty. The uterus contracted well; and the patient recovered favorably.

The foetus was near the full size. Its abdomen was distended with fluid; the skin dark coloured; the cuticle easily detached. Some time before labour the patient had ceased to feel the movements of the child: she was only conscious that it "fell about" in the womb. She knew that it was dead. She had had one living child some years ago ; but several succeeding pregnancies have terminated in the same manner as the one now described. In one labour it was said that the placenta was morbidly adherent, and had to be detached by the hand, much hæmorrhage attending. 
The placenta in this instance was free from decomposition. Its bulk was considerable; it had been a good deal torn in extraction. The greater part presented to the naked eye a healthy appearance. In parts, however, there were whitish glistening masses, interspersed in the ordinary red structure. In some parts, also, the tissue was soft, and easily lacerable. In two different parts there was a mass of firm coagulated blood, evidently of a date anterior to the labour. The cord was dark-coloured, flaccid, and distended with turbid serum.

The microscopical examination of the white portions revealed the same form of fatty degeneration as that described in my former paper-viz., the villi were mostly exsanguine, opaque, thickly studded with spherules of oil; the chorion was thickened, and destitute of nuclei. Similar appearances were also observed; although to a loss marked degree, in portions of the placenta which, to the naked eye, appeared to be healthy.

There were two complications in this case, which render it difficult to determine what share the fatty degeneration of the placenta had in producing the death of the foetus and the hæmorrhage. The placenta was partially attached to the os uteri, and a part morbidly adherent to the walls of the uterus. This morbid adhesion and the soft, lacerable texture might indicate some previous inflammatory action; and there may appear to be some ground for supposing that the fatty degeneration was the consequence of this inflammatory action. The partial attachment of the placenta to the os uteri was probably the cause of the two masses of firm coagulated blood, the result of a partial separation some time prior to the labour. The further separation of the placenta from the os was almost certainly the cause of the hæmorrhage at the commencement of labour. The condition of the child and the state of the cord clearly show that all fœtal circulation had ceased for some time prior to labour. The condition of the placenta observed after expulsion and the hæmorrhage show that considerable vascular connection had been kept up between 
parts of the placenta and the uterus. The effusion of fluid in the peritonæum of the fotus might lead to the conjecture that disease of the fœtus had been the cause of its death.

In the presence of so many complications, I am anxious not to draw any forced conclusions, and not to attribute the hæmorrhage and the death of the fœtus to any one pathological condition to the exclusion of the rest. But, upon taking into consideration the loss of several fœtuses in succession, and how much this circumstance is in accordance with the history of most of the cases of fatty degeneration of the placenta I have met with, I am not disposed to reject altogether the opinion that a similar cause may have been in operation in this instance.

Case vi.-On the 10th of June, 1852, Mr. Humby brought me another placenta. A young lady, in excellent health, was delivered, after a very easy labour, of her first child. There was no hæmorrhage. The child was putrid, the cuticle easily detaching. It had reached the size of maturity. She had observed no symptom indicating the death of the child.

The placenta was very small; the weight $7 \frac{1}{8}$ ounces. It was divided by deep sulci into three principal lobes, and three or four smaller ones. One large lobe and a smaller one were pale, and showed no mark of recent connection with the uterus. Another of the larger lobes had more of the aspect of normal placenta. It had a pale reddish colour, a pulply feel, and there were several fresh venous coagula hanging out of the semi-circular openings on the decidual surface. The rest of the placenta was of a character intermediate between those of the portions above described. The substance of every part was tender, softer than natural, lacerable. It was generally pale and greasy-looking. There was no consolidation in any part.

The membranes were green from commencing putrefaction. The cord was flaccid, and infiltrated with reddish 
serum. There was no change of a putrefactive kind in the substance of the placenta.

Under the microscope, the villi were observed to separate readily; but none were discovered perfectly healthy. In some the nuclei of the chorion and of the vessels were perfect in form, and contained only a few granules. The extremities of many villi were enlarged, and contained much fatty matter in the shape of minute molecules and large spherules or droplets (see fig. 1), giving the extremities, when viewed with a low power, a dark nodulated appearance. The chorion and coats of the vessels were generally studded with oil-granules. The general colour was dull yellow. No blood was discovered in the villi with the altered extremities. In many of the others some blood was seen. ${ }^{1}$

The above changes were observed in every part of the placenta; but they were most advanced in that lobe which showed no mark of recent vascular connection with the uterus. Generally the change of structure was most marked near the maternal surface.

I infer, from the examination of the placenta, that in this case the progress of the degeneration of the placenta had cut off the embryo. The presence of blood in many of the villi which were less altered in structure indicated that there had been a fotal circulation at no remote period; and the evident vascular connection of the maternal placenta with the uterus leads to the same conclusion. The reason why there was no hæmorrhage may be more conveniently discussed hereafter.

Case vir.-On the 1st of September, 1851, my colleague, Mr. Forbes, sent me a placenta for inspection, with the

1 This condition resembles very closely that described by Dr. Franz Kilian. In the majority of instances, the fatty matter assumes the form of granules; but in some cases, it is observed in both forms. In no instance, however, have we observed fatty matter in the form of droplets alone. Wherever the droplets have been noticed in the villi, the coats of the villi have been affected by the granular form of degeneration 
following statement:-A lady was delivered of a dead fœus at about five months from the beginning of gestation. She had had hæmorrhage almost from the commencement. This was her third miscarriage. The fatus had attained the development proper to the fifth month of gestation.

The placenta was fresh. It had not been immersed in any fluid. The foetal surface, as seen through the membranes, generally presented a glistening pale-yellow appearance, resembling fat. The maternal surface was deeply divided by sulci, as is mostly observed in the immature placenta. More than half had a pale-yellow glistening fatty appearance. This was more marked in the superficial portion than in the deeper portions exposed by section. It cut and tore like placental tissue, but differed in being comparatively pale and bloodless. The above description applies to by far the larger portion of the placenta. But there was a second portion, consisting of one lobe, quite distinct from the rest, of a darker colour, and which cut like flesh. A section of this showed engorgement of blood, extending to extravasation. There was also a third portion, which differed from the rest. Along the margin for nearly half the circumference the colour -was darker and the consistence firmer than that of the general mass. There was the appearance of recent effusion of blood and fibrine.

Of the first, or larger portion of the placental mass, one lobe, which was white and almost perfectly exsanguine, exhibited, under the microscope, universal fatty degeneration in the extremities of the villi, which were dilated and misshapen from the presence of the deposit. Two other lobes of this portion, which exhibited the like alteration in many of the villi, but to a less extent, were of a light pink colour. The villi contained a small quantity of blood.

In the second portion, consisting of a distinct lobe, the villi were much distended with blood; some were varicose or irregularly dilated. There was also the same granular alteration as in the first portion, but to a less extent. This portion was intensely congested. 
The third, or marginal portion, appeared consolidated or hepatised. Under the microscope, fragments of villi were seen, in some of which the same granular alteration, as in the first portion, was observed. But the chief part of the substance was composed of effused fibrine, itself degenerating into granular matter.

I have little doubt that this case was one of primary granular degeneration of the villi, leading to the death of the fœtus and abortion. The villi in every part were affected. The engorgement with blood and the varicose appearance of the villi of the second portion would seem to warrant the inference that an unusual effort had been made in this lobe to compensate for the almost complete cessation of circulation in the rest of the placenta. The hepatised appearance and the effusion of fibrine in the third, or marginal, portion were probably the result of a similar excess of action at an earlier period. Possibly they might have been the result of the giving way of the diseased coats of the villi under the additional strain imposed upon them by the imperfect functional capacity of the main portion of the organ. In the main portion there was no evidence of the presence of anything but the ordinary tissues of the placenta, in a state of more or less advanced granular degeneration.

Case vili.-On the 8th of October, 1852, Mr. R. R. Robinson, of Camberwell, forwarded me a portion of placenta, and subsequently communicated the following history. Mrs. - aged 31, a delicate lady, had been the subject of disease of the spine some years ago, which was cured without deformity. She was subject to a short hacking cough; but no disease of the lungs could be detected. She had been married four years. Eleven months after marriage she was delivered of a still-born child after a sevenmonths' pregnancy. Fourteen months after her first confinement she was again delivered of a still-born child after a gestation of six months and a half. She was very weak for a long time afterwards. For the last two years she had 
always a red tongue, and was subject to diarrhœa. In May, 1851, she had an attack of abdominal inflammation, from which she recovered so far as to feel better than for a long time previously. In February last she became pregnant again, and went on very well till the 8th of June, when, having gone out in a carriage, she was much shaken; suddenly some hæmorrhage occurred, attended with uterine pain. In the night this returned. For some weeks she kept in bed, and then was removed to the sofa. Under mild nourishment and the use of steel she derived considerable benefit. On the lst of October Mr. Robinson was requested to see her. She complained of feeling very faint; and not having felt the child for a day or two, she was fearful it was dead. By the use of ammonia, the faintness went off, and she thought she felt the child. On the morning of the 6th, however, she complained of violent pain in the back and sides, and decided labour-pains came on, but subsided. They returned on the following day; a gush of blood came, and a continued draining ensued. The membranes were immediately ruptured, when a large quantity of liquor amnii, not fetid, escaped. The pains increased; there was no further hæmorrhage; and the child was born in twenty minutes. It was a male of seven months' growth; it had evidently been dead some time, as the cuticle peeled off. The placenta quickly followed; the uterus contracted well. Some clots followed: the blood was dark. The cord was thick, red, and gelatinous. The urine was unaffected by heat or nitric acid.

The placenta was rather small, easily torn, and soft. The torn surface was very pale, moist, and shreddy. The portion sent to me by Mr. Robinson was of a uniform dull opaque yellow fatty appearance. It presented on two or three points of the uterine surface marks of recently severed vessels, indicating a limited connection with the uterus. The texture was not at all consolidated; it was generally bloodless. Under the microscope, the villi were seen to be mostly exsanguine. The nuclei along the margin of the chorion were visible, but dotted with granules. The chorion 
was not detached from the vessels, but was generally affected with granular degeneration. Numerous extremities of villi exhibited the black opaque granular aggregations observed in Case vI, and represented in fig. 1. As in that case, the villi showing the nodular aggregations contained no blood.

The appearance of the child and the placenta, and the history of the case, place it beyond a doubt that the child had been dead at least ten days before birth. The presence of blood in the villi least affected, and the recent connection of the maternal placenta to the uterus, led me to infer that the child had been cut off by the advancing degeneration of the foetal portion of the placenta. The hæmorrhage which occurred on the 8th of June-that is, in the fifth month of pregnancy-after succussion, was in all probability owing to partial alteration of the placenta, which rendered it readily liable to partial detachment. There is no reason to suppose it to have been owing to abnormal position.

The impaired health of the patient, as bearing upon the state of nutrition, is worthy of remark.

CASE IX.- On the 17th of June, 1852, a patient of the Western General Dispensary, aborted at about the tenth week of gestation. She ascribed the abortion to emotion consequent upon her mother's death. She had been treated for dyspepsia previously. There was a good deal of hæmorrhage. The ovum came away easily. She had noticed nothing to lead her to suppose she was going to abort.

The decidua and chorion were undoubtedly of an earlier date than ten weeks. I conjectured, from their appearance, that development had been arrested at about the seventh week. The decidua had, however, retained some vascular connection with the uterus to the last. No embryo was discovered. The decidua was very much thickened, and somewhat infiltrated with recent blood. On the uterine surface the oblique vascular openings were distinct; but it 
was pale, and had a fatty appearance. Magnified 420 diam., the cells of the decidua were well marked: most had a caudate elongation at either end. By no amount of laceration could these cells be separated from the fibrous membrane in which they were imbedded. The caudase elongations seemed to interlace with each other, and to effect a firm hold upon the membrane. Numerous granules of oil were seen in the cells. The fotal surface of the decidua presented similar appearances; but the fatty change was less extensive.

The villi of the chorion, viewed with the naked eye, were of a dull yellowish-white colour. They did not freely float out in water. They presented numerous nodular enlargements at the extremities. With an inch glass these enlargements were seen more clearly: they looked like pear. shaped bodies on very narrow stalks, and greatly resembled minute hydatids of the chorion which have shrunk from being kept a day or two. With a quarter-inch glass these enlargements were found to contain granular fat; they were opaque, some quite dark, not always regular in shape, but mostly pyriform. The villi generally presented the nuclei, if not entire, not quite destroyed. Granular fat was abundant throughout. It was clear that the degeneration of the chorion was more extensive than that of the decidua.

The pathological history of this case may admit of some discussion. Whether there is any relation between the peculiar conformation of the villi and the granular degeneration is a matter of doubt. It must also be difficult to determine whether the deviations of form and the alteration of structure of the chorion preceded or followed the destruction of the embryo. But there is little ground to hesitate in concluding that the degeneration of the chorion preceded that of the decidua. This slow progress of the disorganisation of the decidua - an essentially uterine structure-will account for the continued vascular connection with the uterus, and the retention of the ovum for a period of three weeks or more after the destruction of the embryo. 
I think this is the most convenient place to make a few observations upon the singular conformation of the villi in this case. Vrolik has reproduced a plate from Seiler, with the following description:- "Flocci secundum Seiler à 5â vel 6tâ graviditatis hebdomade, vesiculis terminati, que dein evanescunt, sed nonnunquam abnormi ratione permanent et tunc isti spuriæ graviditatis speciei ansam dant, quam molam botryoidam vel hydaticam dicunt. Non opus est monere etiam hac periodo nulla adhuc in chorii floccis formata esse vasa." On comparing the figure of Seiler with the appearance of the chorion in this case, a striking resemblance was observed. It was figured by Seiler and is referred to by Vrolik as the normal form of the chorion at an early period. Désormeaux has described a similar appearance, and attributes the origin of hydatids to the persistence of these apparently vesicular enlargements of the chorion. That the chorion figured by Seiler was diseased is quite certain, and I am disposed to believe it had undergone fatty degeneration. ${ }^{1}$ The chorion is evidently thickened and partially detached; these are changes characteristic of fatty degeneration. It will be interesting to compare this figure of Seiler's with fig. 2, illustrating the next case.

CASE X.-On May 14, 1852, I was called on to see Mrs. $\mathrm{H}-$, a somewhat delicate but healthy lady, who had borne two healthy children at the full period. She had symptoms of abortion at a very early period of gestation. She had been in her usual health on the 10th. For three days she had rather profuse hæmorrhage, which came on without any known cause. She had constant pains; coagula passed at intervals, and there was continual draining. On the second day a portion of chorion, covered with shaggy villi, was passed. On the third day another portion of chorion, with villi covered with minute hydatidiform cysts. Shortly afterwards a portion of decidua, slightly ecchymosed, but otherwise seemingly healthy, came away. No trace of embryo was discovered.

' See Seiler's Plate, fig. 2. 
A little hæmorrhage still continued. Shortly afterwards, as she was being lifted out of bed, more hæmorrhage occurred, and a mass as large as a small orange came away. This mass consisted of decidua infiltrated with recent coagulated blood, and chorion with numerous cysts. Still there was no embryo. After this the hæmorrhage ceased, and no further portions of membranes were observed. The patient went on favorably until the 18th, when, having sat at an open window, she had a stiff neck, acute pains in the left groin, and stiffness in the left leg. She was unable to move the left leg without increasing the pain in the groin and left pelvic region. There was considerable febrile movement and constipation. Leeches relieved the local symptoms; a dose of calomel brought away a large quantity of fæces. On the $22 \mathrm{~d}$ she was much distressed by cough. Auscultation revealed no affection of the chest whatever. I have witnessed in other cases of abortion a most distressing irritative cough supervene, when there had been no symptom of the kind previously, and when every care had been taken to avoid catching cold. It appears to me that the cough, in these instances, is to be ascribed to irritation propagated from the uterine mucous membrane, and that it is analogous to the cough excited by irritation of the mucous membranes of other parts. In this particular case, no relief was afforded by the usual remedies, but the application of galvanism to the chest was attended by the most marked beneficial effects. Mrs. H- gradually recovered her strength.

Examination of parts of the ovum.-A portion of the chorion was entirely free from hydatiginous degeneration. When this portion was viewed with a strong lens, the villi looked of a dull, opaque, yellowish-white. They were more rigid than healthy villi. Some villi were pure white. Some of the ends of the villi were thickened, nodular, and misshapen. Under the microscope, the vascular structure of the villi precisely resembled the villi of the mature placenta in their general character. Where the structures had not undergone much change, the nuclei in the chorion along 
the margin were distinct; but more or less deposition of minute oil spherules was general throughout the structure of the villi. In some, where the change was more advanced, and where the chorion yet remained covering the vessels, it was considerably thickened, opaque, and of a yellowish tinge; the nuclei had disappeared, or nearly so, and the membrane was studded with oil spherules. In parts, the chorion, rendered brittle by change of structure, had become detached from the vessels, which were seen here and there partly denuded, with portions of broken chorion adhering in the form of portions of tube (see fig. 2). When the vessels were completely bare, their walls were discovered to be also affected by fatty degeneration. The vessels contained no blood.

The concurrence of hydatidinous and fatty degeneration in this case is interesting. The progress of either affection would have sufficed to destroy the embryo and lead to abortion; but, judging from the universality of the fatty degeneration and the generally exsanguine condition of the villi, I am disposed to consider this as the chief cause. The decidua was healthy, and had evidently maintained its connection with the uterus to the last. This circumstance, which was satisfactorily ascertained, serves to fix the cause of the abortion in the chorion. A point worthy of observation, is the very early period (probably not more than six weeks) at which fatty degeneration may be developed in the villi.

In connection with this case I will hazard the suggestion, that fatty degeneration may be the process by which those villi of the chorion, whose function becomes superseded at an early stage by the concentration and development of the placenta upon one portion, become atrophied and disappear.

The cases now observed, I believe, furnish illustrations of the influence of fatty degeneration in producing abortion, by rendering the villi of the chorion or placenta unfitted for their office of maintaining the nutrition of the embryo. They also illustrate the relation of this affection to hæmor- 
rhage. I would especially point to Case $x$ as an example of abortion brought about by this disease proceeding to an advanced stage in the villi of the chorion at a very early period of gestation, and before the formation of a distinct placenta; and Case IX might probably be adduced as a similar instance. Case vir is an example of the disease leading to the death of the fotus, and subsequent expulsion of the ovum in the fifth month. Case Iv is an example similar to the preceding, the death of the embryo and abortion occurring at about the end of the fifth month. In Case viIr, Mr. Robinson's, and in Cases I and II in my first paper, the death of the embryo seems to have occurred in the seventh month of gestation. The case of Dr. Franz Kilian exhibits death of the fœtus and expulsion at the end of eight months. Cases $\mathbf{v}$ and vi afford examples of destruction of the embryo in the last month of gestation. It thus appears that fatty degeneration may cause the destruction of the embryo, and consequent abortion, at any period, from the earliest to the latest, of gestation. That the fatty degeneration of the placenta was really the cause of abortion in most of the foregoing cases, appears to me to be established by the following considerations :

In the first place, on referring to the histories of the cases related, it will be observed that in no case was there any sign of decomposition of the placenta.

Secondly, the distinct indications of continued vascular connection between the placenta and the uterus up to the moment of detachment and expulsion.

Thirdly, the various degrees of progress of the degenera. tion in different portions of the same placenta, some portions being far gone towards disorganisation, whilst in others the villi remained comparatively healthy - to the naked eye entirely so-although exhibiting, under the microscope, evidence of the same change in an early stage: these portions still showing, by the presence of blood within the vessels, a sure indication that they had recently fulfilled their allotted functions.

Fourthly, the obvious commensurate diminution of the 
vascular connection between placenta and uterus with the progress of the affection, especially exemplified in Case iv.

Fifthly, the almost constant evidence of the death of the fotus having taken place at some definite but not remote period, usually about fourteen days, before expulsion; and the improbability of this affection of the placentas having commenced and attained the very advanced condition observed within that limited period.

Sixthly, there is the analogical argument derived from the better known pathology of fatty degeneration in other organs, as in the muscular structure of the vessels, heart, in the liver, in the kidneys, and in the blood-vessels. The circumstances under which this destructive change is observed in these instances preclude all doubt that it arises during the life of the individual, and that sooner or later the resulting disorganisation terminates in death. Is it reasonable to conclude that the placenta is the only organ in which identical appearances of structure can be produced as a post-mortem change?

That a particular form of disorganisation, or rather metamorphosis of a fatty character may take place after the death of the placenta, is beyond a doubt. I have myself recorded a case illustrating the fact in the Appendix to my first paper. But the appearances exhibited in this undoubted example of post-mortem granular metamorphosis, are essentially distinct from those observed in all the other cases, and represented in the figures accompanying this and the former paper. One character alone will serve to contrast the fatty degeneration of the living placenta with the granular metamorphosis of the dead organ. In the latter, the change of structure is universal and uniform, affecting alike the chorion and the decidua, the whole extent of the vessels as well as their extremities. To my mind the widely-different characters of the two forms of granular change observed, furnish the most absolute of all the proofs in support of the conclusion, that in the cases now described the structural changes took place during the life of the placenta.

In relation to the question of the influence of fatty 
degeneration of the placenta in bringing about abortion, I think it of great interest to analyse the steps by which the detachment and expulsion of the embryo and secundines are usually effected in those cases in which the embryo perishes prematurely in the uterus. I have referred to the fact that symptoms indicating the death of the fœtus mostly precede its expulsion by some definite period. An examination of the histories of many cases of premature labour, in which the child was born dead, recorded by Mauriceau, Lee, Crosse, and others, fully bears out this proposition. When the fœtus perishes, the first effect is necessarily the arrest of the fœtal circulation. The fœtal blood no longer flowing through the umbilical capillaries of the placenta, the stimulus to the afflux of blood to the maternal portion of the placenta is lost, and the utero-placental circulation soon ceases also. This is the first step. The next step consists in the gradual atrophy of the placenta, of the utero-placental vessels, and the decidua, which still tend to preserve the adhesion of the ovum to the uterus. Why a certain connection is kept up between the placenta and the uterus some time after the placenta as an organ subservient to the nutrition of the embryo has become useless, may, I believe, be accounted for as follows: The fœtal portion of the placenta perishes with the embryo; but the maternal portion, the decidua, is essentially an uterine structure, and tends for some time longer to preserve its relation to the uterus. This is especially remarkable in cases of early abortion, where the cause appears to have resided in the chorion or the embryo. At the very early period of gestation the connection of the decidua with the uterus is of the most intimate kind, and it is altogether distinct from the chorion. In such a case, a dead ovum may be retained in the uterus for a longer time than in the case of death of the fœtus at a more advanced period of gestation. Case ix may be taken as an illustration of this: the separation of the decidua was probably not effected in less than three weeks.

Whilst the bonds of attachment between the placenta and the uterus are becoming thus gradually impaired, and the 
detachment of the placenta is being prepared, important changes are going on in the uterus to prepare for the expulsion of the blighted ovum. During the growth of the ovum the developmental stimulus attracts blood in abundance to the uterus. But when the fotus has perished, and in the course of retrogressive cessation of action the placenta has next become a dead mass, blood is no longer attracted to the uterus. When growth ceases, atrophy begins. In all probability that change which Mr. Rainey has so well described as taking place in the uterus after normal parturition, begins as soon as the contained embryo dies. The muscular element undergoing fatty degeneration gradually recedes. The uterus tends slowly to contract upon itself; its capacity to diminish. So soon as the walls of the uterus have so far collapsed as to press upon the contained ovum, the diastaltic function is excited, and active contractions -labour-pains-complete the detachment, and effect expulsion.

There is an interesting confirmation of this theory. In the case of twin-pregnancy it occasionally happens that one fotus perishes at an early period of gestation, whilst the other lives on, and the whole may be retained till the full term. In these cases the dead ovum is not cast off, because the developmental activity of the uterus is kept up by the living embryo. Cruveilhier has related an interesting example of this nature, and he has figured the two placentas connected with the dead and the living fotuses. The one connected with the dead is represented as yellowish in colour, of a tubercular appearance, very compact and atrophied, and it was evident that for a long time all adhesion had been destroyed. I refer to this description for the double purpose of remarking, that, notwithstanding the atrophy of the placenta, and the absence of all adhesion, it was still retained; and also of suggesting the extreme probability, that the condition of the placenta described and figured by Cruveilhier was in reality that of post-mortem granular metamorphosis resembling the case described in the Appendix to my paper in the 34th volume of the 'Transactions.' 
I will now consider the relation of fatty degeneration of the placenta to hæmorrhage. On referring to the histories of the cases related, it will be observed that in some, hæmorrhage occurred prior to the accession of labour; and that, in others, there was a singular immunity from hæmorrhage throughout. In cases I, IV, VII, IX, $x$, hæmorrhage took place at different periods of gestation, or on the accession of labour. In cases II, $v$, and vilI, no hæmorrhage was observed.

I think I am justified by further experience in adhering to the explanation of the mode in which hæmorrhage is caused by fatty degeneration of the placenta given in my first communication. In many cases the affection proceeds with very unequal rapidity in different portions of the placenta. Some lobes may be far gone, while others do not to the naked eye present any obvious departure from the normal structure. In the altered portions two remarkable conditions may be observed. First : they differ from the normal placenta in consistency; they are no longer spongy and yielding, but of more or less solidity. Secondly, the vascular channels between the placenta and uterus are more or less completely obliterated. The consequence of these two conditions is a great disposition to detachment at the diseased points. If the detachment were strictly limited to the diseased points, where little or no vascular connection with the uterus remains, there would be no hæmorrhage. But this can seldom be the case: the detachment of the diseased lobes is almost sure to entail partial detachment of portions still maintaining a freer vascular connection, and thus hæmorrhage will result. This event may be the immediate occasion of the expulsion of the ovum; or, on the other hand, it may be arrested for a time, and recur several times before labour.

In some cases fatty degeneration seems to invade the entire decidual surface of the placenta with great uniformity. In such cases the consistency of the placenta not only remains equal throughout, but may be soft and lacerable; and there is no portion more disposed than the rest to become detached from the uterus. At a certain stage of the 
progress of the disease, the embryo will have perished, and all fœtal-placental circulation will have ceased. The great cause of vascular activity in the placenta will be at an end; and any vascular connection between the uterus and placenta still remaining, must be confined to such a sluggish circulation as may suffice to preserve a modified nutrition in the placental tissue. But the placenta is essentially a caducous organ ; and when its proper function has terminated, it tends rapidly to break off. The fatty degeneration of the placenta probably goes on at an accelerated ratio after the death of the embryo, the vascular intercourse between placenta and uterus becomes rapidly less and less, until at a period seldom protracted beyond fourteen days, the decidua, the immediate bond of uniou, is so altered as to have become no longer fitted to preserve cohesion with the uterus, and the vessels which tended to unite the placenta to the uterus, are so far obliterated, that when the placenta drops off, as an etiolated leaf from a plant, not a drop of blood may flow. But although the vascular intercourse between the placenta and the uterus at the period of scission may be reduced to a very inconsiderable extent, I have not witnessed a case in which it was altogether arrested. A few recent coagula may always be seen in the severed utero-placental vessels, although no evidence of blood may be detected in the fotal portion of the placenta.

It would be foreign to the purpose of this paper to enter into any discussion as to the general pathology of fatty degeneration; but I wish to submit a few remarks specially bearing upon the relation of this disease to the placenta. By far the most interesting question, and at the same time the most obscure, is the origin of this affection. It is obvious that, in the improvement of our knowledge of the causes and conditions which lead to the development of fatty degeneration, our hope of discovering rational and successful prophylactic measures depends. It is reasonable to suppose that the primary condition determining fatty degeneration in the placenta may arise in the mother or in the embryo. I will pass over the consideration of the 
strictly maternal sources with a simple reference to the marked influence of pregnancy in altering the constitution of the blood-sometimes seriously impairing its quality - and the consequent hazard of defective nutrition both in mother and embryo.

The circumstances which lead to abortion through a primary influence upon the fotus have been greatly overlooked; while the influence of external circumstances acting upon the mother has been in a corresponding degree exaggerated. In the particular instance of fatty degeneration of the placenta it is also possible to attribute an undue importance to the agency of maternal causes. It seems highly probable that an imperfection in, or loss of, developmental force in the embryo may be the frequent occasion of degeneration of the placenta. It is impossible not to recognise the embryo, from the moment of impregnation, as an independent living organism; and it is not altogether fanciful to represent the new being as finding in the uterus a nidus and the pabulum requisite for its nutrition. If the developmental force originally imparted to the germ be defective,-whether the defect arise from the maternal or the spermatic element,-at a more or less advanced period of gestation, the power of assimilation and nutrition in the embryo may flag; and this failing power of assimilation and nutrition in the embryo may be regarded as the immediate occasion of the degeneration of the placenta. This view is strengthened by the fact, clearly established by the investigations Dr. Hassall and myself have made, that this degenerative process begins in, and causes, the most destructive changes in the villi of the chorion. ${ }^{1}$

Reflecting on the important part which fatty degeneration plays in bringing about the restoration of the uterus after parturition to its normal condition, and upon

1 The hypothesis here advanced appears to me strictly in accordance with the views of Professor Owen, on the perpetuation of the "spermatic force " throughout different stages of development of the same individual, or of its propagation through a series of different individuals. 
other similar phenomena in the history of this affection, I was at an early period of my researches led to conjecture that the placenta might be prepared for being cast off from the uterus by a similar process. The observation that the change is usually, if not always, most marked at the decidual surface, supports this conjecture. I have already hinted that the superfluous villi of the chorion of the early ovum may be removed in this way. In order to ascertain how far this view may apply to the mature placenta, I have examined a considerable number of healthy placentas expelled at the full term. The presence of a certain amount of fat is constantly observed; but the observations hitherto made do not authorise the conclusion that the fall of the placenta is prepared for by a process of fatty degeneration.

The descriptions of the microscopical appearances in the cases related in this and the former paper clearly point to an altered condition of the elementary structures of the placenta similar to that condition of the heart, liver, kidney, and muscles, which is expressed by the term "fatty degeneration." I have used this term in reference to the placenta precisely in the sense which it is understood to convey when applied to other organs. I have thought this explanation necessary in order to distinguish the affection which it has been my object to describe from other changes in the placenta which might be, and indeed have been, a source of confusion. The products of inflammation-effusions of fibrine and coagula - it is well known are prone to undergo a process of fatty conversion. Such are frequently found in the placenta; and I have had numerous specimens of this character brought to me under the belief that they were instances of fatty degeneration of the placenta. Fibrinous deposits, solid, pale-yellow, and, when seen through the fœetal membranes, bearing a close resemblance to masses of fat, are commonly observed on the fœtal surface of the placenta. Sometimes this deposit is so extensive as to spread over the entire foetal superficies. These effusions are observed in placentas expelled at the full term of gestation, the children being born alive and 
healthy. They do not appear to impede the circulation through the placental vessels; but in some cases I have witnessed, the quantity of effused fibrine was so great in the proximity of the large vessels gathering to constitute the umbilical trunks, that $I$ have been surprised that these vessels have escaped pressure, or even obliteration, from the great contraction to which effused fibrine is liable. In connection with the form of effused fibrine to which I am now referring, I have not detected any evidence of inflammatory action. The placental tissue in the immediate vicinity has been perfectly normal. I am disposed to believe that fibrine may be poured out into the placenta as the simple consequence of that excessive proportion of fibrine which is characteristic of the blood during pregnancy.

In concluding, I may be permitted to remark that the carefully-observed facts related in this and my former paper, tend to prove that fatty degeneration of the placenta is far from being an uncommon event. That it has hitherto escaped observation is to be attributed to the neglect of the microscope in investigating the abnormal conditions of that organ. Alterations of structure in the elementary tissues of the placenta cannot be recognised without the light of that instrument; and, on the other hand, the gross appearances to the naked eye frequently lead to the most erroneous conclusions. I have already remarked, that placentas have been submitted to me as examples of fatty degeneration which, under the microscope, exhibited the villi in a perfectly healthy state. On the other hand, I confidently believe that the real alteration in many placentas, described by various authors as examples of scirrhous disease, of atrophy, or of tuberclè, might have been shown to be fatty degeneration, had the morbid portions been subjected to microscopical analysis. 
Postscript, Feb. 16, 1853.

CASE XII.-I beg permission to report yet another Case which has occurred since my paper was placed in the hands of the Secretary. It serves to fill up what many may consider to have been a void in the evidence supplied by the cases hitherto reported.

On the 6th February, 1853, a stout adipose woman was delivered of a healthy child, strong and of average size, after a natural labour. She is a patient of the Paddington Infirmary. Dr. Mackenzie had caused the placenta to be examined, because he had, on a former occasion, observed a fatty placenta in a woman who was unusually stout and inclined to obesity.

The placenta was above the average size; a great proportion appeared healthy. Along the circumference was a thick dense fibrinous-looking deposit. There were three or four nodules or masses of degenerated placenta of the size of a walnut on the maternal surface. One of these nodules, especially examined, was of a glistening whitish-yellow aspect, and exsanguine. Under the microscope it was found to consist entirely of villi undergoing granular degeneration; in parts the granules or spherules of oil were numerous, and the chorion investing the vessels more or less broken up. The villi in the vicinity of this nodule, which to the naked eye appeared healthy, were also affected, but to a less extent. The villi in other parts were healthy.

It seems difficult to attribute the commencing degeneration of the placenta in this case to mal-nutrition, or to any other state of the fœtus, which was born alive and strong. The alteration was limited in extent, and no doubt commenced late in pregnancy. Had it begun at an earlier period, it might have had time to invade a large portion of the organ, and have cut off the child before the natural epoch of parturition.

This case appears to me to prove conclusively that fatty degeneration of the placenta may arise independently of 
death or disease of the embryo. This point established, it is unnecessary to show that when this degradation of structure has invaded a large proportion of the placenta, the organ must be rendered unfit for the performance of its functions, and that the foetus must perish in consequence.

This case supplies the last link that was wanting in the chain of evidence, to confirm the leading propositions I have advanced in reference to the pathological value of fatty degeneration of the placenta. 


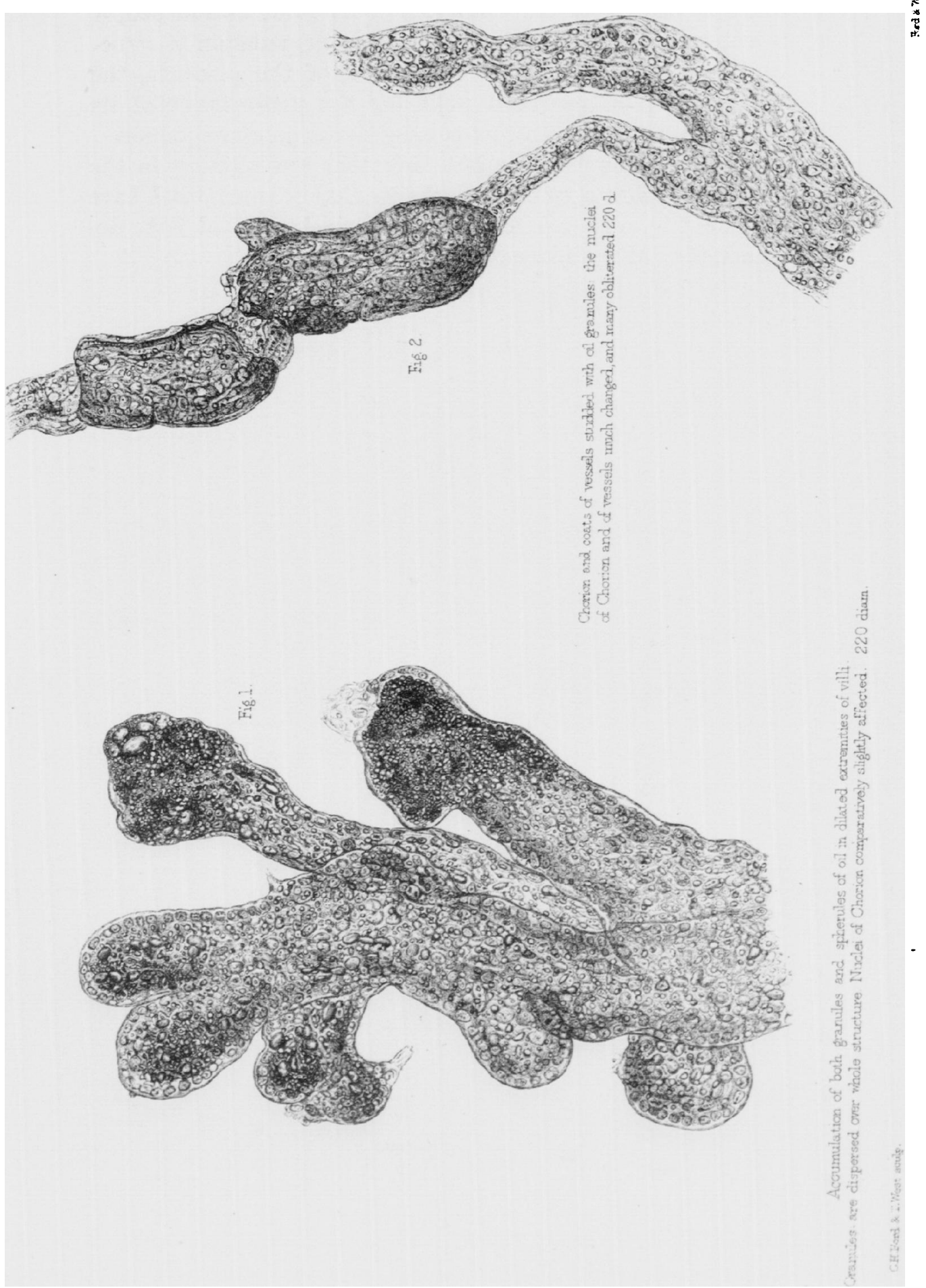

